\title{
Explicit and Energy-Conserving Constraint Energy Minimizing Generalized Multiscale Discontinuous Galerkin Method for Wave Propagation in Heterogeneous Media
}

\author{
Siu Wun Cheung* Eric T. Chung ${ }^{\dagger} \quad$ Yalchin Efendiev $^{\ddagger} \quad$ Wing Tat Leung ${ }^{\S}$
}

September 3, 2020

\begin{abstract}
In this work, we propose a local multiscale model reduction approach for the timedomain scalar wave equation in a heterogenous media. A fine mesh is used to capture the heterogeneities of the coefficient field, and the equation is solved globally on a coarse mesh in the discontinuous Galerkin discretization setting. The main idea of the model reduction approach is to extract dominant modes in local spectral problems for representation of important features, construct multiscale basis functions in coarse oversampled regions by constraint energy minimization problems, and perform a Petrov-Galerkin projection and a symmetrization onto the coarse grid. The method is expicit and energy conserving, and exhibits both coarse-mesh and spectral convergence, provided that the oversampling size is appropriately chosen. We study the stability and convergence of our method. We also present numerical results on the Marmousi model in order to test the performance of the method and verify the theoretical results.
\end{abstract}

\section{Introduction}

In this paper, we consider a local multiscale model reduction approach for the scalar wave equation. Let $\Omega \subset \mathbb{R}^{d}$ be a bounded spatial domain. For the sake of simplicity, we focus our discussion on two-dimensional cases in this paper, though the extension to the three-dimensional case shall be straightforward. We consider the following scalar wave equation

$$
\frac{\partial^{2} u}{\partial t^{2}}=\operatorname{div}(\kappa \nabla u)+f \text { in }[0, T] \times \Omega,
$$

where $f(x, t)$ is a given source term. The problem is subject to the homogeneous Dirichlet boundary condition $u=0$ on $[0, T] \times \partial \Omega$, and initial conditions $u(x, 0)=u_{0}(x)$ and $u_{t}(x, 0)=$ $v_{0}(x)$ in $\Omega$. We assume that the coefficient field $\kappa$ is a heterogeneous coefficient field with contrast $\kappa_{0} \leq \kappa \leq \kappa_{1}$. Due to the heterogeneities in the coefficient field, numerical discretization requires a very fine grid mesh in order to capture all the heterogeneities in the medium properties, which potentially makes the numerical solutions on the fine grid become prohibitively expensive.

\footnotetext{
*Department of Mathematics, Texas A\&M University, College Station, TX 77843, USA (tonycsw2905@math.tamu.edu)

${ }^{\dagger}$ Department of Mathematics, The Chinese University of Hong Kong, Shatin, New Territories, Hong Kong SAR, China (tschung@math.cuhk.edu.hk)

¥Department of Mathematics \& Institute for Scientific Computation (ISC), Texas A\&M University, College Station, Texas, USA (efendiev@math.tamu.edu)

$\S$ Institute for Computational Engineering and Sciences, The University of Texas at Austin, Austin, Texas, USA (wleungo@ices.utexas.edu)
} 
Extensive research effort had been devoted to developing numerical solvers for solving multiscale problems on the coarse grid, which is typically much coarser than the fine grid, such as numerical homogenization approaches 45, 151, 43, 2, Multiscale Finite Element Methods (MsFEM) 34, 29, 6, 28, 10, 39, Variational Multiscale Methods (VMS) 36, 37, 38, 5, 40, 49, Heterogeneous Multiscale Methods (HMM) [22, 1, 23, 33, 3] and and Generalized Multsicale Finite Element Methods (GMsFEM) 24, 21, 14, 11, 13, 19. In numerical homogenization approaches, effective properties are computed for formulating the global problem on the coarse grid. However, these approaches are limited to the cases when the medium properties possess scale separation. On the other hand, multiscale methods construct of multiscale basis functions which are responsible for capturing the local oscillatory effects of the solution. Once the multiscale basis functions are constructed, coarse-scale equations are formulated. Moreover, fine-scale information can be recovered by the coarse-scale coefficients and mutliscale basis functions. In recent years, multiscale methods in the discontinuous Galerkin (DG) framework have been investigated 229, 4, 47, 25, 32, 30, 17, 20. In these approaches, unlike conforming finite element formulations, multiscale basis functions are in general discontinuous on the coarse grid, and stabilization or penalty terms are added to ensure well-posedness of the global problem.

In many state-of-the-art mutliscale methods, such as MsFEM, VMS and HMM, there is one basis function per local coarse region to handle the effects of local heterogeneities. However, for more complex multiscale problems, each local coarse region contains several high-conductivity regions and multiple multiscale basis functions are required to represent the local solution space. GMsFEM is developed to allow systematic enrichment of the coarse-scale space with fine-scale information and identify the underlying low-dimensional local structures for solution representation. The main idea of GMsFEM is to extract local dominant modes by carefully designed local spectral problems in coarse regions, and the convergence of the GMsFEM is related to eigenvalue decay of local spectral problems. For a more detailed discussion on GMsFEM, we refer the readers to 27, 24, 26, 21, 16, 11, 31, 7, 9, 46, 50, 48, 8, and the references therein. Through the design of local spectral problems, our method results in the minimal degree of freedom in representing high-contrast features. In particular, 15] considered an application of GMsFEM on scalar wave equations. On the other hand, several multiscale methods with mesh convergence are developed. 44, 41, 42. This idea can be adopted for multiscale model reduction techniques for achieving both spectral and mesh convergence [35, 18, 12, 7, 8].

In this paper, we present the Constraint Energy Minimizing Generalized Multiscale Discontinuous Galerkin Method (CEM-GMsDGM). Our method results in coarse-scale equations in an interior penalty discontinuous Galerkin (IPDG) discretization setting. The method is expicit and energy conserving, and exhibits both coarse-mesh convergence and spectral convergence. The advantages of the method are verified both theoretically and numerically. The model reduction approach possesses of two key ingredients. The first main ingredient is the local spectral problems in each coarse block for identification of multiscale test basis functions. The low-energy dominant modes, which are eigenvectors corresponding to small eigenvalues of local spectral problems, are used as multiscale test basis functions, as well as for further construction of the second ingredient of our method, which is a set of multiscale trial basis functions. Each of the test basis functions sets up an independent orthongonality constraints and uniquely defines a corresponding multiscale trial basis function. The multiscale trial basis functions will then be used for a coarse-scale represenation of the numerical solution. We remark that the local spectral problems and the constraint energy minimization problems are carefully designed and supported by our analysis.

The paper is organized as follows. In Section 2 we will introduce the notions of grids, and essential discretization details such as DG finite element spaces and IPDG formulation on the coarse grid. The details of the proposed method, including the construction of basis functions and the corresponding systems of linear equations, will be presented in Section 3 . The stability and the convergence of the method will be analyzed in Section 4 Numerical results will be provided in Section 5. Finally, a conclusion will be given in Section 6 . 


\section{IPDG formulation}

We are now going to introduce some notions of coarse and fine meshes. We start with a usual partition $\mathcal{T}^{H}$ of $\Omega$ into finite elements, which does not necessarily resolve any multiscale features. The partition $\mathcal{T}^{H}$ is called a coarse grid and a generic element $K$ in the partition $\mathcal{T}^{H}$ is called a coarse element. Moreover, $H>0$ is called the coarse mesh size. We let $N_{c}$ be the number of coarse grid nodes and $N$ be the number of coarse elements. We also denote the collection of all coarse grid edges by $\mathcal{E}^{H}$. We perform a refinement of $\mathcal{T}^{H}$ to obtain a fine grid $\mathcal{T}^{h}$, where $h>0$ is called the fine mesh size. It is assumed that the fine grid is sufficiently fine to resolve the solution. An illustration of the fine grid and the coarse grid and a coarse element are shown in Figure 1

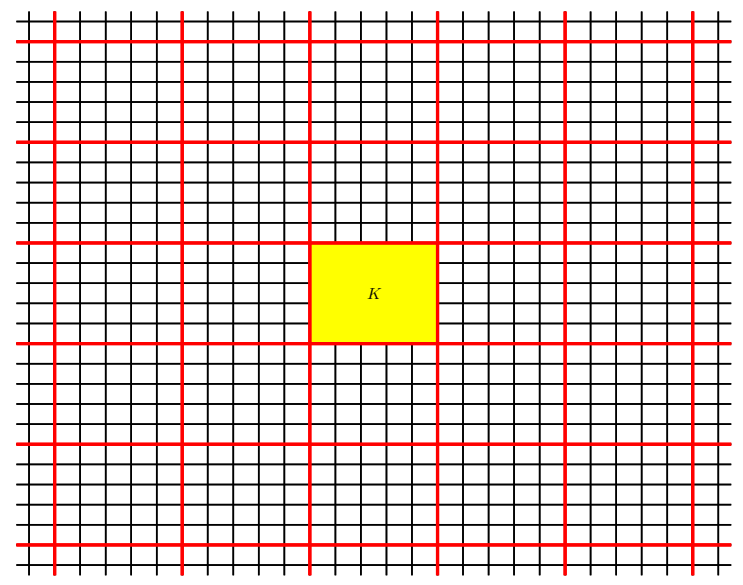

Figure 1: An illustration of the fine grid and the coarse grid and a coarse element.

We are now going to discuss the discontinuous Galerkin (DG) discretization and the interior penalty discontinuous Galerkin (IPDG) global formulation. For the $i$-th coarse block $K_{i}$, we let $V_{h}\left(K_{i}\right)$ be the conforming bilinear elements defined on the fine grid $\mathcal{T}^{h}$ in $K_{i}$. The DG approximation space is then given by the space of coarse-scale locally conforming piecewise bilinear fine-grid basis functions, that is,

$$
V_{h}=\oplus_{i=1}^{N} V_{h}\left(K_{i}\right)
$$

We remark that functions in $V_{h}$ are continuous within coarse blocks, but discontinuous across the coarse grid edges in general. We define the symmetric IPDG bilinear form $a_{D G}$ by:

$$
\begin{aligned}
a_{D G}(v, w)= & \sum_{K \in \mathcal{T}^{H}} \int_{K} \kappa \nabla v \cdot \nabla w d x-\sum_{E \in \mathcal{E}^{H}} \int_{E}\left\{\kappa \nabla v \cdot n_{E}\right\} \llbracket w \rrbracket d \sigma \\
& -\sum_{E \in \mathcal{E}^{H}} \int_{E}\left\{\kappa \nabla w \cdot n_{E}\right\} \llbracket v \rrbracket d \sigma+\frac{\gamma}{h} \sum_{E \in \mathcal{E}^{H}} \int_{E} \bar{\kappa} \llbracket v \rrbracket \llbracket w \rrbracket d \sigma,
\end{aligned}
$$

where $\gamma>0$ is a penalty parameter and $n_{E}$ is a fixed unit normal vector defined on the coarse edge $E \in \mathcal{E}^{H}$. Note that, in (3), the average and the jump operators are defined in the classical way. Specifically, consider an interior coarse edge $E \in \mathcal{E}^{H}$ and let $K^{+}$and $K^{-}$be the two coarse grid blocks sharing the edge $E$, where the unit normal vector $n_{E}$ is pointing from $K^{+}$to $K^{-}$. For a piecewise smooth function $G$ with respect to the coarse grid $\mathcal{T}^{H}$, we define

$$
\begin{aligned}
\{G\} & =\frac{1}{2}\left(G^{+}+G^{-}\right), \\
\llbracket G \rrbracket & =G^{+}-G^{-},
\end{aligned}
$$


where $G^{+}=\left.G\right|_{K^{+}}$and $G^{-}=\left.G\right|_{K^{-}}$. Moreover, on the edge $E$, we define $\bar{\kappa}=\left(\kappa_{K^{+}}+\kappa_{K^{-}}\right) / 2$, where $\kappa_{K^{ \pm}}$is the maximum value of $\kappa$ over $K^{ \pm}$. For a coarse edge $E$ lying on the boundary $\partial \Omega$, we define $\{G\}=\llbracket G \rrbracket=G$, and $\kappa=\kappa_{K}$ on $E$, where we always assume that $n_{E}$ is pointing outside of $\Omega$. We also use the notation $(\cdot, \cdot)_{L^{2}(D)}$ to denote the standard $L^{2}(D)$ scalar product for a subdomain $D \subset \Omega$.

Using the IPDG spatial discretization, we derive the semi-discrete scheme: find $u_{h}(t, \cdot) \in V_{h}$ such that

$$
\left(\frac{\partial^{2} u_{h}}{\partial t^{2}}, w\right)_{L^{2}(\Omega)}+a_{D G}\left(u_{h}, w\right)=(f, w)_{L^{2}(\Omega)} \text { in }[0, T] \times \Omega
$$

where the initial data is projected onto the finite element space $V_{h}$ by: find $u_{h}(\cdot, 0), \frac{\partial u_{h}}{\partial t}(\cdot, 0) \in V_{h}$ such that for all $w \in V_{h}$,

$$
\begin{aligned}
\left(u_{h}(\cdot, 0), w\right)_{L^{2}(\Omega)} & =\left(u_{0}, w\right)_{L^{2}(\Omega)}, \\
\left(\frac{\partial u_{h}}{\partial t}(\cdot, 0), w\right)_{L^{2}(\Omega)} & =\left(v_{0}, w\right)_{L^{2}(\Omega)} .
\end{aligned}
$$

\section{Local multiscale model reduction by CEM-GMsFEM}

In this section, we will present our local multiscale model reduction on the IPDG formuation for the wave equation by Constraint Energy Minimizing Generlized Multiscale Finite Element Method. First, we will use the concept of GMsFEM spectral problems to construct our multiscale test basis functions on a generic coarse block $K$ in the coarse grid. Next, we will use the concept of constrained energy minimization to construct our multiscale trial basis functions. Then, we will derive our coarse-scale model with a Petrov-Galerkin projection and a symmetrc formulation. Finally, we present a technique of localization of multiscale trial basis functions on coarse oversampled regions, and which results in an explicit time-marching coarse-scale scheme.

\subsection{Multiscale test functions}

To construct the multiscale test functions, we follow the concept of GMsFEM and perform a multisale model reduction through a local spectral problem on $V_{h}\left(K_{i}\right)$, which is to find a real number $\lambda_{j}^{(i)}$ and a function $\phi_{j}^{(i)} \in V_{h}\left(K_{i}\right)$ such that

$$
a_{i}\left(\phi_{j}^{(i)}, w\right)=\frac{\lambda_{j}^{(i)}}{H^{2}}\left(\phi_{j}^{(i)}, w\right)_{L^{2}\left(K_{i}\right)} \text { for all } w \in V_{h}\left(K_{i}\right),
$$

where $a_{i}$ is a symmetric positive semi-definite bilinear form defined as

$$
a_{i}(v, w)=\int_{K_{i}} \kappa \nabla v \cdot \nabla w d x .
$$

Without loss of generality we shall assume the eigenfunctions are normalized, i.e.

$$
\left(\phi_{j}^{(i)}, \phi_{j^{\prime}}^{(i)}\right)_{L^{2}\left(K_{i}\right)}=\delta_{j, j^{\prime}} \text { for all } 1 \leq j, j^{\prime} \leq L_{i} .
$$

We let $\lambda_{j}^{(i)}$ be the eigenvalues of (77) arranged in ascending order in $j$, and use the first $L_{i}$ eigenfunctions to construct our local multiscale test space

$$
W_{H}^{(i)}=\operatorname{span}\left\{\phi_{j}^{(i)}: 1 \leq j \leq L_{i}\right\} .
$$


We also introduce a local $L^{2}\left(K_{i}\right)$ projection operator $\pi_{i}: V_{h} \rightarrow W_{H}^{(i)}$ onto $W_{H}^{(i)}$ by

$$
\pi_{i}(v)=\sum_{j=1}^{L_{i}}\left(v, \phi_{j}^{(i)}\right)_{L^{2}\left(K_{i}\right)} \phi_{j}^{(i)} \text { for all } v \in V_{h} .
$$

The global multiscale test space $W_{H}$ is then defined as the sum of these local multiscale test spaces

$$
W_{H}=\oplus_{i=1}^{N} W_{H}^{(i)} .
$$

Since the coarse blocks are disjoint, the multiscale test functions form an $L^{2}(\Omega)$ orthonormal basis function for $W_{H}$, i.e.

$$
\left(\phi_{j}^{(i)}, \phi_{j^{\prime}}^{\left(i^{\prime}\right)}\right)_{L^{2}(\Omega)}=\delta_{i, i^{\prime}} \delta_{j, j^{\prime}} \text { for all } 1 \leq j \leq L_{i}, 1 \leq j^{\prime} \leq L_{i^{\prime}} \text { and } 1 \leq i, i^{\prime} \leq N,
$$

and the global $L^{2}(\Omega)$ projection operator $\pi: V_{h} \rightarrow W_{H}$ onto $W_{H}$ is then naturally defined by $\pi=\sum_{i=1}^{N} \pi_{i}$.

\subsection{Multiscale trial functions}

Next, we construct our global multiscale trial functions space $V_{H}$ using the concepts of constraint energy minimization. Given a multiscale test basis function $\psi_{j}^{(i)}$, where $1 \leq j \leq L_{i}$ and $1 \leq i \leq$ $N$, the global multiscale trial basis function $\psi_{j}^{(i)} \in V_{h}$ is defined as the solution of the following constrained energy minimization problem

$$
\psi_{j}^{(i)}=\underset{\psi \in V_{h}}{\operatorname{argmin}}\left\{a_{D G}(\psi, \psi): \pi(\psi)=\phi_{j}^{(i)}\right\} .
$$

By introducing a Lagrange multiplier, the minimization problem (14) is equivalent to the following variational problem: find $\psi_{j}^{(i)} \in V_{h}$ and $\mu_{j}^{(i)} \in W_{H}$ such that

$$
\begin{aligned}
a_{D G}\left(\psi_{j}^{(i)}, \psi\right)+\left(\psi, \mu_{j}^{(i)}\right)_{L^{2}(\Omega)} & =0 \text { for all } \psi \in V_{h}, \\
\left(\psi_{j}^{(i)}-\phi_{j}^{(i)}, \mu\right)_{L^{2}(\Omega)} & =0 \text { for all } \mu \in W_{H} .
\end{aligned}
$$

We use the global multiscale trial basis functions to construct the multiscale trial space, which is defined as

$$
V_{H}^{(\infty)}=\operatorname{span}\left\{\psi_{j}^{(i)}: 1 \leq j \leq L_{i}, 1 \leq i \leq N\right\}
$$

\subsection{Global coarse-scale model}

We derive our fully discrete coarse-scale system by a second-order central difference for temporal discretization. means of Petrov-Galerkin projection of the fine-scale system onto the coarse-scale spaces. Let $N_{T}$ be the number of time steps in the temporal mesh grid and $\tau=T / N_{T}$ be the time step size. At the time instant $t_{n}=n \tau$, we denote the evaluation of the source function $f$ at the time instant $t_{n}$ by $f^{n}$, and an approximation of the solution $u\left(\cdot, t_{n}\right)$ by $u_{H}^{n}$. The coarse-scale model which reads: for $n \geq 1$, find $u_{H}^{n+1} \in V_{H}^{(\infty)}$ such that

$$
\left(\frac{u_{H}^{n+1}-2 u_{H}^{n}+u_{H}^{n-1}}{\tau^{2}}, w\right)_{L^{2}(\Omega)}+a_{D G}\left(u_{H}^{n}, w\right)=\left(f^{n}, w\right)_{L^{2}(\Omega)} \text { for all } w \in W_{H},
$$


where the initial data is projected onto the finite element space $V_{H}^{(\infty)}$ by: find $u_{h}^{0}, u_{h}^{1} \in V_{H}^{(\infty)}$ such that for all $w \in V_{H}^{(\infty)}$,

$$
\begin{aligned}
& \left(u_{H}^{0}, w\right)_{L^{2}(\Omega)}=\left(u_{0}, w\right)_{L^{2}(\Omega)}, \\
& \left(u_{H}^{1}, w\right)_{L^{2}(\Omega)}=\left(u_{0}+\tau v_{0}+\frac{\tau^{2}}{2} f^{0}, w\right)_{L^{2}(\Omega)}-\frac{\tau^{2}}{2} a_{D G}\left(u_{H}^{0}, w\right) .
\end{aligned}
$$

Next, we are going to present a symmetric formulation of (17) on $V_{H}^{(\infty)}$. By a simple dimensionality argument, it is easy to see that $\left.\pi\right|_{V_{H}^{(\infty)}}: V_{H}^{(\infty)} \rightarrow W_{H}$ is an isomorphism. Moreover, for any $v \in V_{H}^{(\infty)}$, using the fact that

$$
(v-\pi(v), w)_{L^{2}(\Omega)}=0 \text { for all } w \in W_{H},
$$

it is straightforward to verify that

$$
a_{D G}(\psi, v-\pi(v))=0 \text { for all } \psi \in V_{H}^{(\infty)} .
$$

Combining all these facts, (17) can be rewritten as

$$
b\left(\frac{u_{H}^{n+1}-2 u_{H}^{n}+u_{H}^{n-1}}{\tau^{2}}, w\right)+a_{D G}\left(u_{H}^{n}, w\right)=b\left(f^{n}, w\right) \text { for all } w \in V_{H}^{(\infty)},
$$

where the bilinear form $b$ is defined as

$$
b(v, w)=(\pi(v), \pi(w))_{L^{2}(\Omega)} .
$$

\subsection{Localization of multiscale trial functions}

One major drawback of the above constructive procedure is that the multiscale trial functions have to be defined by solving a global problem. Based on our analysis in 8 , the global multiscale trial basis function $\psi_{j}^{(i)}$ exhibits an exponential decay property, where the value is very small in locations which are far away from the block $K_{i}$. The allows us to construct localized multiscale basis functions on suitably enlarged oversampled domain without a significant increase of approximation error. More precisely, we denote by $K_{i, m}$ an oversampled domain formed by enlarging the coarse grid block $K_{i}$ by $m$ coarse grid layers, of which an illustration shown in Figure 2

We introduce the subspaces $V_{h}\left(K_{i, m}\right)$, which contains restriction of fine-scale basis functions in $V_{h}$ on the oversampled domain $K_{i, m}$, and $W_{H}\left(K_{i, m}\right)$, which is the sum of the local multiscale test space over the coarse blocks in the oversampled domain $K_{i, m}$, i.e.

$$
W_{H}\left(K_{i, m}\right)=\oplus_{i^{\prime}} \subset K_{i, m} W_{H}^{\left(i^{\prime}\right)} .
$$

The localized multiscale trial basis function $\psi_{j, m}^{(i)} \in V_{h}$ is then defined as the solution of the following constrained energy minimization problem

$$
\psi_{j, m}^{(i)}=\underset{\psi \in V_{h}\left(K_{i, m}\right)}{\operatorname{argmin}}\left\{a_{D G}(\psi, \psi): \pi(\psi)=\phi_{j}^{(i)}\right\} .
$$

Using the method of Lagrange multiplier, the minimization problem (24) is equivalent to the following variational problem: find $\psi_{j, m}^{(i)} \in V_{h}\left(K_{i, m}\right)$ and $\mu_{j, m}^{(i)} \in W_{H}\left(K_{i, m}\right)$ such that

$$
\begin{aligned}
a_{D G}\left(\psi_{j, m}^{(i)}, \psi\right)+\left(\psi, \mu_{j, m}^{(i)}\right)_{L^{2}\left(K_{i, m}\right)} & =0 \text { for all } \psi \in V_{h}\left(K_{i, m}\right), \\
\left(\psi_{j, m}^{(i)}-\phi_{j}^{(i)}, \mu\right)_{L^{2}\left(K_{i, m}\right)} & =0 \text { for all } \mu \in W_{H}\left(K_{i, m}\right) .
\end{aligned}
$$




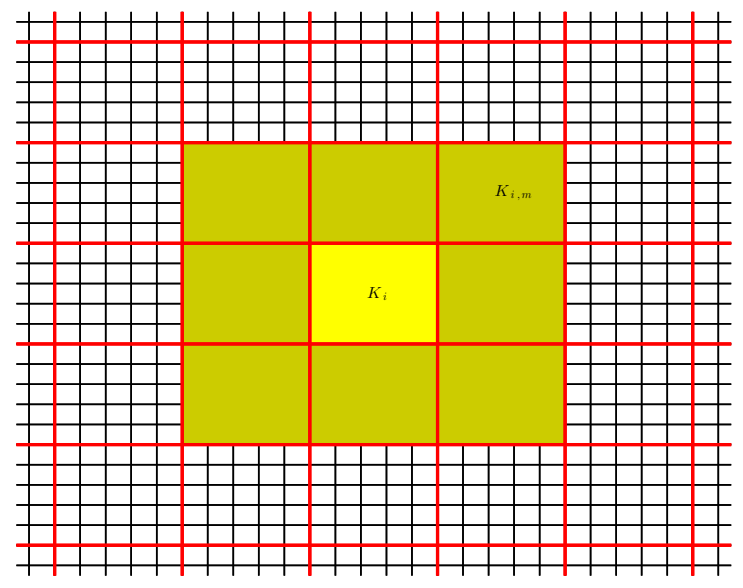

Figure 2: An illustration of an oversampled domain formed by enlarging $K_{i}$ with 1 coarse grid layer.

The localized multiscale trial basis functions then used to define the localized multiscale trial space, i.e.

$$
V_{H}^{(m)}=\operatorname{span}\left\{\psi_{j, m}^{(i)}: 1 \leq j \leq L_{i}, 1 \leq i \leq N\right\}
$$

Finally, our localized coarse-grid model reads: for $n \geq 1$, find $u_{H}^{n+1} \in V_{H}^{(m)}$ such that

$$
b\left(\frac{u_{H}^{n+1}-2 u_{H}^{n}+u_{H}^{n-1}}{\tau^{2}}, w\right)+a_{D G}\left(u_{H}^{n}, w\right)=b\left(f^{n}, w\right) \text { for all } w \in V_{H}^{(m)},
$$

where the initial data is projected onto the finite element space $V_{H}^{(m)}$ by: find $u_{H}^{0}, u_{H}^{1} \in V_{H}^{(m)}$ such that for all $w \in V_{H}^{(m)}$,

$$
\begin{aligned}
& \left(u_{H}^{0}, w\right)_{L^{2}(\Omega)}=\left(u_{0}, w\right)_{L^{2}(\Omega)}, \\
& \left(u_{H}^{1}, w\right)_{L^{2}(\Omega)}=\left(u_{0}+\tau v_{0}+\frac{\tau^{2}}{2} f^{0}, w\right)_{L^{2}(\Omega)}-\frac{\tau^{2}}{2} a_{D G}\left(u_{h}^{0}, w\right) .
\end{aligned}
$$

\subsection{Linear system associated with localized coarse-scale model}

We end this section by the derivation of the resultant coarse-scale system of linear equations with a fixed global enumeration of nodal indices and multiscale basis function indices. Denote by $\mathbf{M}$ and $\mathbf{A}$ the matrix representation of the $L^{2}(\Omega)$ scalar product and the IPDG bilinear form $a_{D G}$ with respect to the fine-scale nodal basis functions in $V_{h}$. Letting $\mathbf{U}_{h}(\cdot, t)$ be the column vector consisting of coordinate representation of $u_{h}(\cdot, t) \in V_{h}$ with respect to fine-scale nodal basis functions in $V_{h}$, the fine-scale system (5) can be written as

$$
\mathbf{M} \frac{\partial^{2} \mathbf{U}_{h}}{\partial t^{2}}+\mathbf{A} \mathbf{U}_{h}=\mathbf{F}
$$

It is trivial to see that, for any $v \in V_{H}^{(m)}$, the coefficient representation of $v$ by the multiscale trial basis functions is given by

$$
v=\sum_{i=1}^{N} \sum_{j=1}^{L_{i}}\left(v, \phi_{j}^{(i)}\right)_{L^{2}(\Omega)} \psi_{j, m}^{(i)},
$$


which implies the coordinate representations of $v$ with respect to the multiscale trial basis functions $\left\{\psi_{j, m}^{(i)}\right\}$ and $\pi(v)$ with respect to the multiscale trial basis functions $\left\{\phi_{j}^{(i)}\right\}$ coincide exactly. With a fixed global enumeration of nodal indices and multiscale basis function indices, let $\Phi$ and $\Psi$ be the matrix assembled from the column vector consisting the coordinate representation of $\phi_{j}^{(i)}$ and $\psi_{j, m}^{(i)}$ with respect to the fine-scale nodal basis functions in $V_{h}$. Letting $\mathbf{U}_{H}^{n}$ be the column vector consisting of coordinate representation of $u_{H}^{n} \in V_{H}^{(m)}$ with respect to the basis functions $\left\{\psi_{j, m}^{(i)}\right\}$, the coarse-scale system (27) can be written as

$$
\boldsymbol{\Phi}^{\top} \mathbf{M} \boldsymbol{\Phi} \mathbf{U}_{H}^{n+1}=\boldsymbol{\Phi}^{\top} \mathbf{M} \boldsymbol{\Phi}\left(2 \mathbf{U}_{h}^{n}-\mathbf{U}_{h}^{n-1}\right)+\tau^{2}\left(\boldsymbol{\Phi}^{\top} \mathbf{F}^{n}-\boldsymbol{\Psi}^{\top} \mathbf{A} \boldsymbol{\Psi} \mathbf{U}_{h}^{n}\right) .
$$

However, noting that the multiscale test basis functions $\left\{\phi_{j}^{(i)}\right\}$ are $L^{2}(\Omega)$ orthonormal, we have $\boldsymbol{\Phi}^{\top} \mathbf{M} \boldsymbol{\Phi}=\mathbf{I}$, and result in an explicit local multiscale model reduction scheme

$$
\mathbf{U}_{H}^{n+1}=\left(2 \mathbf{U}_{H}^{n}-\mathbf{U}_{H}^{n-1}\right)+\tau^{2}\left(\boldsymbol{\Phi}^{\top} \mathbf{F}^{n}-\Psi^{\top} \mathbf{A} \boldsymbol{\Psi} \mathbf{U}_{h}^{n}\right) .
$$

Once the explicit scheme is used to obtain the coarse-scale coefficients at a final time step, a multiscale approximation is obtained by downscaling with $\mathbf{U}_{h}^{n} \approx \mathbf{\Psi} \mathbf{U}_{H}^{n}$.

\section{$4 \quad$ Stability and convergence analysis}

In this section, we will analyze the stabiility of proposed localized coarse-grid model (27) and obtain an error estimate when the coarse-grid solution is compared with the fine-grid solution obtained from (5). Unless otherwise specified, the constants are generic and independent of mesh size and number of oversampling layers. First we define a norm on $V_{h}$ by

$$
\|w\|_{a}^{2}=\sum_{K \in \mathcal{T}^{H}} \int_{K} \kappa|\nabla w|^{2} d x+\frac{\gamma}{h} \sum_{E \in \mathcal{E}^{H}} \int_{E} \bar{\kappa} \llbracket w \rrbracket^{2} d \sigma .
$$

In our analysis, we will make use of following coercivity and continuity results on the IPDG bilinear form, provided that the penalty parameter is sufficiently large.

Lemma 1. [15] With a sufficiently large $\gamma>0$, there holds

$$
\begin{aligned}
\left|a_{D G}(v, w)\right| & \leq 2\|v\|_{a}\|w\|_{a} \text { for all } v, w \in V_{h}, \\
a_{D G}(v, v) & \geq \frac{1}{2}\|v\|_{a}^{2} \text { for all } v \in V_{h} .
\end{aligned}
$$

Moreover, there exists $\beta>0$ such that

$$
\|v\|_{L^{2}(\Omega)}^{2} \geq \beta \kappa_{1}^{-1} h^{2} a_{D G}(v, v) \text { for all } v \in V_{h} .
$$

With these estimates from the IPDG bilinear form, we now establish an inverse Poincaré inequality on the multiscale test function space. We will need two fundamental results about from [8]. The first result is a stability estimate about the projection operator $\pi$.

Lemma 2. [8] With a smallness assumption on the fine grid mesh $h>0$, there exists $C>0$ such that for any $w_{H} \in W_{H}$, there exists a function $v \in C^{0}(\Omega) \cap V_{h}$ such that

$$
\pi(v)=w_{H}, \quad\|v\|_{a}^{2} \leq D \kappa_{1} H^{-2}\left\|w_{H}\right\|_{L^{2}(\Omega)}^{2}, \quad \operatorname{supp}(v) \subseteq \operatorname{supp}\left(w_{H}\right) .
$$


The second result states that the global multiscale test functions have a decay property and their values are small outside a suitably large oversampled domain, which in turn justifies the localization of the multiscale test functions.

Lemma 3. [8] With the above assumptions, there exists $C, C^{\prime}>0$ such that

$$
\left\|\psi_{j}^{(i)}-\psi_{j, m}^{(i)}\right\|_{a}^{2} \leq C\left(1+C^{\prime}\right)^{-m}\left(1+\Lambda^{-1}\right) \kappa_{1} H^{-2}\left\|\phi_{j}^{(i)}\right\|_{L^{2}\left(K_{i}\right)}^{2},
$$

where $\Lambda$ the smallest eigenvalue which is excluded in the local spectral problem (7), i.e.

$$
\Lambda=\min _{1 \leq i \leq N} \lambda_{L_{i}+1}^{(i)} .
$$

The eigenvalues are related to the contrast in the medium properties. In applying to high contrast media, the eigenvalues exhibit sharp decay and we can simply pick the first few eigenfunctions and ensure the smallest eigenvalue excluded is sufficiently large. The norm relations in the multiscale space is related to the eigenvalue decay in the local spectral problems, which accounts for the approximation error by the multiscale finite element space. First of all, for any $v \in V_{h}$, we have

$$
\|(I-\pi)(v)\|_{L^{2}(\Omega)}^{2} \leq 2 \Lambda^{-1} H^{2} a_{D G}(v, v) .
$$

Moreover, we have the following inverse inequality.

Lemma 4. With the above assumptions, there exists $\beta>0$ such that

$$
\|\pi(v)\|_{L^{2}(\Omega)}^{2} \geq \beta \kappa_{1}^{-1} H^{2} a_{D G}(v, v) \text { for all } v \in V_{H}^{(m)} .
$$

Proof. For any $v \in V_{H}^{(m)}$, we define

$$
\begin{aligned}
& \widetilde{v}=\sum_{i=1}^{N} \sum_{j=1}^{L_{i}}\left(v, \phi_{j}^{(i)}\right)_{L^{2}(\Omega)} \psi_{j}^{(i)}, \\
& \eta=\sum_{i=1}^{N} \sum_{j=1}^{L_{i}}\left(v, \phi_{j}^{(i)}\right)_{L^{2}(\Omega)} \mu_{j}^{(i)} .
\end{aligned}
$$

Then we have $\pi(v)=\pi(\widetilde{v})$. By the definition of global multiscale test functions (15), for any $\psi \in V_{h}$, we have

$$
a_{D G}(\widetilde{v}, \psi)+(\pi(\psi), \eta)_{L^{2}(\Omega)}=0 .
$$

By Lemma 2, we take $w \in C^{0}(\Omega) \cap V_{h}$ such that

$$
\pi(w)=\eta, \quad\|w\|_{a}^{2} \leq D \kappa_{1} H^{-2}\|\eta\|_{L^{2}(\Omega)}^{2}, \quad \operatorname{supp}(w) \subseteq \operatorname{supp}(\eta) .
$$

Taking $\psi=w$ in (42), we have

$$
\|\eta\|_{L^{2}(\Omega)}^{2} \leq 2\|\widetilde{v}\|_{a}\|w\|_{a} \leq 2 D^{\frac{1}{2}} \kappa_{1}^{\frac{1}{2}} H^{-1}\|\widetilde{v}\|_{a}\|\eta\|_{L^{2}(\Omega)}
$$

Taking $\psi=\widetilde{v}$ in (42), we have

$$
\frac{1}{2}\|\widetilde{v}\|_{a}^{2} \leq\|\pi(\widetilde{v})\|_{L^{2}(\Omega)}\|\eta\|_{L^{2}(\Omega)} .
$$

Combining these estimates, we have

$$
\|\widetilde{v}\|_{a} \leq 4 D^{\frac{1}{2}} \kappa_{1}^{\frac{1}{2}} H^{-1}\|\pi(v)\|_{L^{2}(\Omega)} .
$$


On the other hand, by Lemma 3, we have

$$
\begin{aligned}
\|\widetilde{v}-v\|_{a}^{2} & \leq C m^{d} \sum_{i=1}^{N}\left\|\sum_{j=1}^{L_{i}}\left(v, \phi_{j}^{(i)}\right)_{L^{2}(\Omega)}\left(\psi_{j}^{(i)}-\psi_{j, m}^{(i)}\right)\right\|_{a}^{2} \\
& \leq C m^{d}\left(1+\Lambda^{-1}\right) \kappa_{1} H^{-2}\left(1+C^{\prime}\right)^{-m} \sum_{i=1}^{N}\left\|\sum_{j=1}^{L_{i}}\left(v, \phi_{j}^{(i)}\right)_{L^{2}(\Omega)} \phi_{j}^{(i)}\right\|_{L^{2}\left(K_{i}\right)}^{2} \\
& =C\left(1+\Lambda^{-1}\right) m^{d}\left(1+C^{\prime}\right)^{-m} \kappa_{1} H^{-2}\|\pi(v)\|_{L^{2}(\Omega)}^{2} .
\end{aligned}
$$

Combining these estimates, we have

$$
\|v\|_{a}^{2} \leq\left(16 D^{2}+C\left(1+\Lambda^{-1}\right) m^{d}\left(1+C^{\prime}\right)^{-m}\right) \kappa_{1} H^{-2}\|\pi(v)\|_{L^{2}(\Omega)}^{2} .
$$

Finally, we note that $m^{d}\left(1+C^{\prime}\right)^{-m}$ is bounded. The proof is complete by taking

$$
\beta^{2}=16 D^{2}+C\left(1+\Lambda^{-1}\right) \sup _{m \geq 1} m^{d}\left(1+C^{\prime}\right)^{-m} .
$$

We are now going to define a discrete total energy which is related to the stability and convergence of our method. Given a sequence of states $v=\left\{v^{n}\right\}_{n=0}^{N_{T}}$, we define the discrete total energy at $t=t_{n+\frac{1}{2}}$ by

$$
\begin{aligned}
E^{n+\frac{1}{2}}(v)=\frac{1}{2} & \left\|\pi\left(\frac{v^{n+1}-v^{n}}{\tau}\right)\right\|_{L^{2}(\Omega)}^{2}-\frac{\tau^{2}}{8} a_{D G}\left(\frac{v^{n+1}-v^{n}}{\tau}, \frac{v^{n+1}-v^{n}}{\tau}\right) \\
& +\frac{1}{2} a_{D G}\left(\frac{v^{n+1}+v^{n}}{2}, \frac{v^{n+1}+v^{n}}{2}\right),
\end{aligned}
$$

which is non-negative under a stability condition. More precisely, if there holds

$$
\rho=\frac{\kappa_{1}^{\frac{1}{2}} \tau}{2 \beta^{\frac{1}{2}} H}<1
$$

then we have the following inequality

$$
E^{n+\frac{1}{2}}(v) \geq \frac{1-\rho^{2}}{2}\left\|\pi\left(\frac{v^{n+1}-v^{n}}{\tau}\right)\right\|_{L^{2}(\Omega)}^{2}+\frac{1}{2} a_{D G}\left(\frac{v^{n+1}+v^{n}}{2}, \frac{v^{n+1}+v^{n}}{2}\right) \geq 0,
$$

due to the result (40) in Lemma 4. The following lemma is the key of proving the stability and convergence of our method.

Lemma 5. For $n \geq 1$, given $r^{n} \in L^{2}(\Omega)$ and $v^{n-1}, v^{n} \in V_{H}^{(m)}$, suppose $v^{n+1} \in V_{H}^{(m)}$ solves

$$
b\left(\frac{v^{n+1}-2 v^{n}+v^{n-1}}{\tau^{2}}, w\right)+a_{D G}\left(v^{n}, w\right)=\left(r^{n}, w\right)_{L^{2}(\Omega)} \text { for all } w \in V_{H}^{(m)} .
$$

Then we have

$$
E^{n+\frac{1}{2}}(v)=E^{\frac{1}{2}}(v)+\tau \sum_{k=1}^{n}\left(r^{k}, \frac{v^{k+1}-v^{k-1}}{2 \tau}\right)_{L^{2}(\Omega)}
$$


Moreover, with the above assumptions, then there exist a $C>0$ such that

$$
E^{n+\frac{1}{2}}(v) \leq C\left(E^{\frac{1}{2}}(v)+\left(\tau R_{1}^{n}+\Lambda^{-\frac{1}{2}} H R_{2}^{n}\right)^{2}\right) .
$$

where

$$
\begin{aligned}
& R_{1}^{n}=\sum_{k=1}^{n}\left\|\pi\left(r^{k}\right)\right\|_{L^{2}(\Omega)}, \\
& R_{2}^{n}=\left\|(I-\pi) r^{1}\right\|_{L^{2}(\Omega)}+\tau \sum_{k=1}^{n-1}\left\|(I-\pi)\left(\frac{r^{k+1}-r^{k}}{\tau}\right)\right\|_{L^{2}(\Omega)}+\left\|(I-\pi) r^{n}\right\|_{L^{2}(\Omega)} .
\end{aligned}
$$

Proof. Taking $w=\frac{v^{n+1}-v^{n-1}}{2 \tau} \in V_{H}^{(m)}$ in (53), we have

$$
\begin{aligned}
& \frac{1}{2 \tau}\left(\left\|\pi\left(\frac{v^{n+1}-v^{n}}{\tau}\right)\right\|_{L^{2}(\Omega)}^{2}-\left\|\pi\left(\frac{v^{n}-v^{n-1}}{\tau}\right)\right\|_{L^{2}(\Omega)}^{2}+a_{D G}\left(v^{n+1}, v^{n}\right)-a_{D G}\left(v^{n}, v^{n-1}\right)\right) \\
& \quad=\left(r^{n}, \frac{v^{n+1}-v^{n-1}}{2 \tau}\right)_{L^{2}(\Omega)} .
\end{aligned}
$$

We observe that

$$
a_{D G}\left(v^{n+1}, v^{n}\right)=a_{D G}\left(\frac{v^{n+1}+v^{n}}{2}, \frac{v^{n+1}+v^{n}}{2}\right)-\frac{\tau^{2}}{4} a_{D G}\left(\frac{v^{n+1}-v^{n}}{\tau}, \frac{v^{n+1}-v^{n}}{\tau}\right) .
$$

Hence, we have

$$
E^{n+\frac{1}{2}}(v)-E^{n-\frac{1}{2}}(v)=\tau\left(r^{n}, \frac{v^{n+1}-v^{n-1}}{2 \tau}\right)_{L^{2}(\Omega)} .
$$

Using a telescoping sum, we obtain (54). To obtain the second result, we rewrite the right hand side of (59) by

$$
\begin{aligned}
\left(r^{n}, \frac{v^{n+1}-v^{n-1}}{2 \tau}\right)_{L^{2}(\Omega)}= & b\left(r^{n}, \frac{v^{n+1}-v^{n-1}}{2 \tau}\right)+\left((I-\pi) r^{n}, \frac{v^{n+1}-v^{n-1}}{2 \tau}\right)_{L^{2}(\Omega)} \\
= & \frac{1}{2}\left(b\left(r^{n}, \frac{v^{n+1}-v^{n}}{\tau}\right)+b\left(r^{n}, \frac{v^{n}-v^{n-1}}{\tau}\right)\right)+ \\
& \frac{1}{\tau}\left((I-\pi) r^{n},(I-\pi)\left(\frac{v^{n+1}+v^{n}}{2}\right)\right)_{L^{2}(\Omega)}- \\
& \frac{1}{\tau}\left((I-\pi) r^{n},(I-\pi)\left(\frac{v^{n}+v^{n-1}}{2}\right)\right)_{L^{2}(\Omega)} .
\end{aligned}
$$


Substituting (60) into (54) and rearranging the indices, we obtain

$$
\begin{aligned}
& E^{n+\frac{1}{2}}(v)=E^{\frac{1}{2}}(v)+\frac{\tau}{2} \sum_{k=1}^{n}\left(b\left(r^{k}, \frac{v^{k+1}-v^{k}}{\tau}\right)+b\left(r^{k}, \frac{v^{k}-v^{k-1}}{\tau}\right)\right)+ \\
&\left((I-\pi) r^{n},(I-\pi)\left(\frac{v^{n+1}+v^{n}}{2}\right)\right)_{L^{2}(\Omega)}-\left((I-\pi) r^{1},(I-\pi)\left(\frac{v^{1}+v^{0}}{2}\right)\right)_{L^{2}(\Omega)}- \\
& \tau \sum_{k=1}^{n-1}\left((I-\pi)\left(\frac{r^{k+1}-r^{k}}{\tau}\right),(I-\pi)\left(\frac{v^{k+1}+v^{k}}{2}\right)\right)_{L^{2}(\Omega)} .
\end{aligned}
$$

Using Cauchy-Schwarz inequality and Young's inequality, we have

$$
\begin{aligned}
E^{n+\frac{1}{2}}(v) \leq E^{\frac{1}{2}}(v)+\max _{0 \leq k \leq n}\left\|\pi\left(\frac{v^{k+1}-v^{k}}{\tau}\right)\right\|_{L^{2}(\Omega)} \tau R_{1}^{n} \\
\quad+\max _{0 \leq k \leq n}\left\|(I-\pi)\left(\frac{v^{k+1}+v^{k}}{2}\right)\right\|_{L^{2}(\Omega)} R_{2}^{n} \\
\leq E^{\frac{1}{2}}(v)+\frac{1-\rho^{2}}{4} \max _{0 \leq k \leq n}\left\|\pi\left(\frac{v^{k+1}-v^{k}}{\tau}\right)\right\|_{L^{2}(\Omega)}^{2}+\frac{\tau^{2}}{1-\rho^{2}}\left(R_{1}^{n}\right)^{2} \\
+\frac{\Lambda}{8 H^{2}} \max _{0 \leq k \leq n}\left\|(I-\pi)\left(\frac{v^{k+1}+v^{k}}{2}\right)\right\|_{L^{2}(\Omega)}^{2}+\frac{2 H^{2}}{\Lambda}\left(R_{2}^{n}\right)^{2} .
\end{aligned}
$$

Using the inequalities (39) and (52), we obtain the desired result.

A direct consequence of Lemma 5 is the following stability result of the coarse-grid solution.

Theorem 6. With the above assumptions, we have the following stability estimate

$$
\left\|\pi\left(\frac{u_{H}^{n+1}-u_{H}^{n}}{\tau}\right)\right\|_{L^{2}(\Omega)}^{2}+a_{D G}\left(\frac{u_{H}^{n+1}+u_{H}^{n}}{2}, \frac{u_{H}^{n+1}+u_{H}^{n}}{2}\right) \leq C\left(E^{\frac{1}{2}}\left(u_{H}\right)+\tau^{2}\left(\sum_{k=1}^{n}\left\|\pi\left(f^{k}\right)\right\|_{L^{2}(\Omega)}\right)^{2}\right)
$$

To proceed with our convergence analysis, we need to define two operators. The first one is a solution map $G_{h}: L^{2}(\Omega) \rightarrow V_{h}$ defined by: for any $g \in L^{2}(\Omega)$, the image $G_{h} g \in V_{h}$ is defined as

$$
a_{D G}\left(G_{h} g, w\right)=(g, w)_{L^{2}(\Omega)} \text { for all } w \in V_{h} .
$$

Second, we define an elliptic projection $P_{H}: V_{h} \rightarrow V_{H}^{(m)}$ by: for any $v \in V_{h}$, the image $P_{H} v \in$ $V_{H}^{(m)}$ is defined as

$$
a_{D G}\left(P_{H} v, w\right)=a_{D G}(v, w) \text { for all } w \in V_{H}^{(m)} .
$$

The approximation error of the DG elliptic projection depends on the eigenvalue decay in the local spectral problems and has a first-order convergence in the coarse mesh size $H$, provided that the discretization follows certain conditions. The proof is very much similar to [8] and is omitted. 
Lemma 7. [8] With the smallest assumptions and the following relation about the size of oversampling region as coarse mesh refines

$$
m=O\left(\log \left(\frac{\kappa_{1}}{H}\right)\right)
$$

there holds

$$
\left\|\left(I-P_{H}\right) G_{h} g\right\|_{a} \leq C \Lambda^{-\frac{1}{2}} H\|g\|_{L^{2}(\Omega)} \text { for all } g \in L^{2}(\Omega) .
$$

It is possible to prove the $L^{2}$ error converges in second order with a duality argument.

Lemma 8. With the above assumptions, there holds

$$
\left\|\left(I-P_{H}\right) G_{h} g\right\|_{L^{2}(\Omega)} \leq C \Lambda^{-1} H^{2}\|g\|_{L^{2}(\Omega)} \text { for all } g \in L^{2}(\Omega) .
$$

Proof. For any $v \in V_{h}$, by Galerkin orthogonality, we have

$$
a_{D G}\left(\left(I-P_{H}\right) v, P_{H} G_{h}\left(I-P_{H}\right) v\right)=0 .
$$

which implies

$$
\begin{aligned}
\left\|\left(I-P_{H}\right) v\right\|_{L^{2}(\Omega)}^{2} & =a_{D G}\left(G_{h}\left(I-P_{H}\right) v,\left(I-P_{H}\right) v\right) \\
& =a_{D G}\left(\left(I-P_{H}\right) G_{h}\left(I-P_{H}\right) v,\left(I-P_{H}\right) v\right) \\
& \leq 2\left\|\left(I-P_{H}\right) G_{h}\left(I-P_{H}\right) v\right\|_{a}\left\|\left(I-P_{H}\right) v\right\|_{a} \\
& \leq C \Lambda^{-\frac{1}{2}} H\left\|\left(I-P_{H}\right) v\right\|_{L^{2}(\Omega)}\left\|\left(I-P_{H}\right) v\right\|_{a},
\end{aligned}
$$

where we have applied the result from Lemma 7 with replacing $g$ by $\left(I-P_{H}\right) v$. In other words,

$$
\left\|\left(I-P_{H}\right) v\right\|_{L^{2}(\Omega)} \leq C \Lambda^{-\frac{1}{2}} H\left\|\left(I-P_{H}\right) v\right\|_{a} .
$$

Applying the result from Lemma 7 again with $v=G_{h} g$, we finish our proof.

Now, with all the tools defined, we are going to estimate the error between the fine-scale solution $u_{h}^{n}=u_{h}\left(t_{n}\right)$ obtained from solving (5) and the coarse-scale solutions $u_{H}^{n}$ obtained from solving (27). We define the quantities

$$
\begin{aligned}
& \varepsilon^{n}=u_{h}^{n}-u_{H}^{n}, \\
& \delta^{n}=u_{H}^{n}-P_{H} u_{h}^{n}, \\
& \theta^{n}=\left(I-P_{H}\right) u_{h}^{n} .
\end{aligned}
$$

We have the following estimates on the error of the elliptic projection.

Lemma 9. With the above assumptions and assuming $f \in C^{4}\left([0, T] ; L^{2}(\Omega)\right)$, there exists $C>0$ such that

$$
\begin{aligned}
\left\|\theta^{n}\right\|_{L^{2}(\Omega)} & \leq C \Lambda^{-1} H^{2}\left(\|g\|_{C\left([0, T] ; L^{2}(\Omega)\right)}+\tau^{2}\left\|\frac{\partial^{2} g}{\partial t^{2}}\right\|_{C\left([0, T] ; L^{2}(\Omega)\right)}\right), \\
\left\|\frac{\theta^{n+1}-2 \theta^{n}+\theta^{n-1}}{\tau^{2}}\right\|_{L^{2}(\Omega)} & \leq C \Lambda^{-1} H^{2}\left(\left\|\frac{\partial^{2} g}{\partial t^{2}}\right\|_{C\left([0, T] ; L^{2}(\Omega)\right)}+\tau^{2}\left\|\frac{\partial^{4} g}{\partial t^{4}}\right\|_{C\left([0, T] ; L^{2}(\Omega)\right)}\right), \\
\left\|\delta^{1}-\delta^{0}\right\|_{L^{2}(\Omega)} & \leq C \tau\left(\Lambda^{-1} H^{2}\left\|\frac{\partial g}{\partial t}\right\|_{C^{0}\left([0, T] ; L^{2}(\Omega)\right)}+\tau^{2}\left\|\frac{\partial^{3} u_{h}}{\partial t^{3}}\right\|_{C^{0}\left([0, T] ; L^{2}(\Omega)\right)}\right),
\end{aligned}
$$

where $g=f-\frac{\partial^{2} u_{h}}{\partial t^{2}}$. 
Proof. First, we observe from the definitions that $u_{h}^{n}=G_{h}\left(g\left(\cdot, t_{n}\right)\right)$. By Taylor's theorem, we have

$$
g\left(\cdot, t_{n}+t\right)=g\left(\cdot, t_{n}\right)+t \frac{\partial g}{\partial t}\left(\cdot, t_{n}\right)+\int_{t_{n}}^{t_{n}+t} s \frac{\partial^{2} g}{\partial t^{2}}(\cdot, s) d s .
$$

Integrating from $t=-\tau$ to $t=\tau$, we have

$$
\left\|g\left(\cdot, t_{n}\right)\right\|_{L^{2}(\Omega)} \leq \frac{1}{2 \tau}\|g\|_{L^{1}\left(t_{n-1}, t_{n+1} ; L^{2}(\Omega)\right)}+\frac{\tau}{2}\left\|\frac{\partial^{2} g}{\partial t^{2}}\right\|_{L^{1}\left(t_{n-1}, t_{n+1} ; L^{2}(\Omega)\right)} .
$$

The first result follows directly from Lemma 8, For the second result, using Taylor's theorem again, we have

$$
g\left(\cdot, t_{n}+t\right)=g\left(\cdot, t_{n}\right)+t \frac{\partial g}{\partial t}\left(\cdot, t_{n}\right)+\frac{t^{2}}{2} \frac{\partial^{2} g}{\partial t^{2}}\left(\cdot, t_{n}\right)+\frac{t^{3}}{6} \frac{\partial^{3} g}{\partial t^{3}}\left(\cdot, t_{n}\right)+\int_{t_{n}}^{t_{n}+t} \frac{s^{3}}{6} \frac{\partial^{4} g}{\partial t^{4}}(\cdot, s) d s .
$$

Taking $t= \pm \tau$, we have

$$
\left\|g\left(\cdot, t_{n+1}\right)-2 g\left(\cdot, t_{n}\right)+g\left(\cdot, t_{n-1}\right)\right\|_{L^{2}(\Omega)} \leq \tau^{2}\left\|\frac{\partial^{2} g}{\partial t}\left(\cdot, t_{n}\right)\right\|_{L^{2}(\Omega)}+\frac{\tau^{3}}{3}\left\|\frac{\partial^{4} g}{\partial t^{4}}\right\|_{L^{1}\left(t_{n-1}, t_{n+1} ; L^{2}(\Omega)\right)} .
$$

The second result now follows from Lemma 8 and replacing $g$ by $\frac{\partial^{2} g}{\partial t^{2}}$ in (75). For the third result, with the same trick, we obtain

$$
\|g(\cdot, \tau)-g(\cdot, 0)\|_{L^{2}(\Omega)} \leq \tau\left\|\frac{\partial g}{\partial t}\right\|_{C\left([0, T] ; L^{2}(\Omega)\right)},
$$

which implies

$$
\left\|\theta^{1}-\theta^{0}\right\|_{L^{2}(\Omega)} \leq C \Lambda^{-1} H^{2} \tau\left\|\frac{\partial f}{\partial t}-\frac{\partial^{3} u_{h}}{\partial t^{3}}\right\|_{C^{0}\left([0, T] ; L^{2}(\Omega)\right)} .
$$

On the other hand, using Taylor's theorem on $u_{h}$, we have

$$
u_{h}(\cdot, \tau)=u_{h}(\cdot, 0)+\tau \frac{\partial u_{h}}{\partial t}(\cdot, 0)+\frac{\tau^{2}}{2} \frac{\partial^{2} u_{h}}{\partial t^{2}}\left(\cdot, t_{n}\right)+\int_{0}^{\tau} \frac{s^{2}}{2} \frac{\partial^{3} u_{h}}{\partial t^{3}}(\cdot, s) d s .
$$

Recalling the definition of $u_{H}^{0}, u_{H}^{1} \in V_{H}^{(m)}$ and taking an $L^{2}(\Omega)$ inner product with $w \in V_{H}^{(m)}$, we observe that

$$
\begin{aligned}
& \left(u_{h}^{0}, w\right)_{L^{2}(\Omega)}=\left(u_{H}^{0}, w\right)_{L^{2}(\Omega)}, \\
& \left(u_{h}^{1}, w\right)_{L^{2}(\Omega)}=\left(u_{H}^{1}, w\right)_{L^{2}(\Omega)}+\int_{0}^{\tau} \frac{s^{2}}{2}\left(\frac{\partial^{3} u_{h}}{\partial t^{3}}(\cdot, s), w\right)_{L^{2}(\Omega)} d s .
\end{aligned}
$$

This yields

$$
\left\|\varepsilon^{1}-\varepsilon^{0}\right\|_{L^{2}(\Omega)} \leq \frac{\tau^{3}}{6}\left\|\frac{\partial^{3} u_{h}}{\partial t^{3}}\right\|_{C^{0}\left([0, T] ; L^{2}(\Omega)\right)} .
$$

The third result then follows from a triangle inequality.

Now, with all the tools defined, we are going to estimate the distance between the coarsesolution solution and elliptic projection. 
Theorem 10. With the above assumptions and assuming $f \in C\left([0, T] ; H^{1}(\Omega)\right)$, we have the following estimate

$$
\left\|\pi\left(\frac{\delta^{n+1}-\delta^{n}}{\tau}\right)\right\|_{L^{2}(\Omega)}^{2}+a_{D G}\left(\frac{\delta^{n+1}+\delta^{n}}{2}, \frac{\delta^{n+1}+\delta^{n}}{2}\right) \leq C\left(\Lambda^{-1} H^{2}+\tau^{2}\right) .
$$

Proof. Recalling the definitions (5) of $u_{h}$ and (27) of $u_{H}$ and noting that $a_{D G}\left(\theta^{n}, w\right)=0$, for any $w \in V_{H}^{(m)}$, we have

$$
b\left(\frac{\delta^{n+1}-2 \delta^{n}+\delta^{n-1}}{\tau^{2}}, w\right)+a_{D G}\left(\delta^{n}, w\right)=\left(r^{n}, w\right)_{L^{2}(\Omega)},
$$

where $r^{n}$ is given by

$$
r^{n}=\pi\left(\frac{\theta^{n+1}-2 \theta^{n}+\theta^{n-1}}{\tau^{2}}\right)+\pi\left(\frac{\partial^{2} u_{h}}{\partial t^{2}}\left(t_{n}, \cdot\right)-\frac{u_{h}^{n+1}-2 u_{h}^{n}+u_{h}^{n-1}}{\tau^{2}}\right)+(I-\pi)\left(f^{n}\right) .
$$

By Lemma 5, we have

$$
\left\|\pi\left(\frac{\delta^{n+1}-\delta^{n}}{\tau}\right)\right\|_{L^{2}(\Omega)}^{2}+a_{D G}\left(\frac{\delta^{n+1}+\delta^{n}}{2}, \frac{\delta^{n+1}+\delta^{n}}{2}\right) \leq C\left(E^{\frac{1}{2}}(\delta)+\left(\tau R_{1}^{n}+\Lambda^{-\frac{1}{2}} H R_{2}^{n}\right)^{2}\right),
$$

where

$$
\begin{aligned}
& R_{1}^{n}=\sum_{k=1}^{n}\left\|\pi\left(\frac{\theta^{k+1}-2 \theta^{k}+\theta^{k-1}}{\tau^{2}}\right)\right\|_{L^{2}(\Omega)}+\sum_{k=1}^{n}\left\|\pi\left(\frac{\partial^{2} u_{h}}{\partial t^{2}}\left(t_{k}, \cdot\right)-\frac{u_{h}^{k+1}-2 u_{h}^{k}+u_{h}^{k-1}}{\tau^{2}}\right)\right\|_{L^{2}(\Omega)}, \\
& R_{2}^{n}=\left\|(I-\pi)\left(f^{1}\right)\right\|_{L^{2}(\Omega)}+\tau \sum_{k=1}^{n-1}\left\|(I-\pi)\left(\frac{f^{k+1}-f^{k}}{\tau}\right)\right\|_{L^{2}(\Omega)}+\left\|(I-\pi)\left(f^{n}\right)\right\|_{L^{2}(\Omega)} .
\end{aligned}
$$

First, by Lemma 1 and Lemma 4, we note that

$$
E^{\frac{1}{2}}(\delta) \leq \frac{1+\rho^{2}}{2}\left\|\pi\left(\frac{\delta^{1}-\delta^{0}}{\tau}\right)\right\|_{L^{2}(\Omega)}^{2}+\left\|\frac{\delta^{1}+\delta^{0}}{2}\right\|_{a}^{2} .
$$

By Lemma 7 and the thired inequality in Lemma 9, we have

$$
E^{\frac{1}{2}}(\delta) \leq C\left(\Lambda^{-1} H^{2}+\tau^{2}\right) .
$$

Next, with the second inequality in Lemma 9 , the first term in $R_{1}^{n}$ can be estimated by

$$
\sum_{k=1}^{n}\left\|\pi\left(\frac{\theta^{k+1}-2 \theta^{k}+\theta^{k-1}}{\tau^{2}}\right)\right\|_{L^{2}(\Omega)} \leq C \tau^{-1} \Lambda^{-1} H^{2}
$$

Similarly, we estimate the second term in $R_{1}^{n}$ using Taylor's expansion

$$
\sum_{k=1}^{n}\left\|\pi\left(\frac{\partial^{2} u_{h}}{\partial t^{2}}\left(t_{k}, \cdot\right)-\frac{u_{h}^{k+1}-2 u_{h}^{k}+u_{h}^{k-1}}{\tau^{2}}\right)\right\|_{L^{2}(\Omega)} \leq C \tau\left\|\frac{\partial^{4} u_{h}}{\partial t^{4}}\right\|_{C\left([0, T] ; L^{2}(\Omega)\right.} .
$$


On the other hand, we estimate the terms in $R_{2}^{n}$ by

$$
\begin{aligned}
\left\|(I-\pi)\left(f^{k}\right)\right\|_{L^{2}(\Omega)} & \leq \Lambda^{-\frac{1}{2}} H\|f\|_{C\left([0, T] ; H^{1}(\Omega)\right)}, \\
\tau \sum_{k=1}^{n}\left\|(I-\pi)\left(\frac{f^{k+1}-f^{k}}{\tau}\right)\right\|_{L^{2}(\Omega)} & \leq C\left\|\frac{\partial f}{\partial t}\right\|_{C\left([0, T] ; L^{2}(\Omega)\right.},
\end{aligned}
$$

Combining all these estimates, we have

$$
\tau R_{1}^{n}+\Lambda^{-\frac{1}{2}} H R_{2}^{n} \leq C\left(\Lambda^{-1} H^{2}+\tau^{2}\right),
$$

which completes the proof.

We complete this section by providing a $L^{2}$ error estimate.

Theorem 11. With the above assumptions, we have the following error estimate

$$
\max _{0 \leq k \leq N_{T}-1}\left\|\frac{\varepsilon^{k+1}+\varepsilon^{k}}{2}\right\|_{L^{2}(\Omega)} \leq C\left(\Lambda^{-1} H^{2}+\tau^{2}\right) .
$$

Proof. We denote $\Delta^{n}=\tau \sum_{k=1}^{n} \delta^{k}$. Using a telescoping sum over (844), we have

$$
b\left(\frac{\delta^{n+1}-\delta^{n}}{\tau}, w\right)-b\left(\frac{\delta^{1}-\delta^{0}}{\tau}, w\right)+a_{D G}\left(\Delta^{n}, w\right)=\tau \sum_{k=1}^{n}\left(r^{k}, w\right)_{L^{2}(\Omega)} .
$$

Taking $w=\Delta^{n+1}-\Delta^{n-1}=\tau\left(\delta^{n+1}+\delta^{n}\right) \in V_{H}^{(m)}$, we imply

$$
\begin{gathered}
\left\|\pi\left(\delta^{n+1}\right)\right\|_{L^{2}(\Omega)}^{2}-\left\|\pi\left(\delta^{n}\right)\right\|_{L^{2}(\Omega)}^{2}+a_{D G}\left(\Delta^{n}, \Delta^{n+1}\right)-a_{D G}\left(\Delta^{n-1}, \Delta^{n}\right) \\
=b\left(\delta^{1}-\delta^{0}, \delta^{n+1}+\delta^{n}\right)+\tau^{2} \sum_{k=1}^{n}\left(r^{k}, \delta^{n+1}+\delta^{n}\right)_{L^{2}(\Omega)} .
\end{gathered}
$$

Using another telescoping sum, we have

$$
\begin{aligned}
& \left\|\pi\left(\delta^{n+1}\right)\right\|_{L^{2}(\Omega)}^{2}+a_{D G}\left(\Delta^{n}, \Delta^{n+1}\right)-\left\|\pi\left(\delta^{1}\right)\right\|_{L^{2}(\Omega)}^{2} \\
& \quad=\sum_{k=1}^{n}\left(b\left(\delta^{1}-\delta^{0}, \delta^{k+1}+\delta^{k}\right)+\tau^{2} \sum_{s=1}^{k}\left(r^{s}, \delta^{k+1}+\delta^{k}\right)_{L^{2}(\Omega)}\right) .
\end{aligned}
$$

We estimate each of the terms of the error identity (97). For the second term on left hand side of (97), we have

$$
\begin{aligned}
a_{D G} & \left(\Delta^{n}, \Delta^{n+1}\right) \\
& =\frac{1}{4}\left(a_{D G}\left(\Delta^{n+1}+\Delta^{n}, \Delta^{n+1}+\Delta^{n}\right)-a_{D G}\left(\Delta^{n+1}-\Delta^{n} \Delta^{n+1}-\Delta^{n}\right)\right) \\
& =a_{D G}\left(\frac{\Delta^{n+1}+\Delta^{n}}{2}, \frac{\Delta^{n+1}+\Delta^{n}}{2}\right)-\frac{\tau^{2}}{4} a_{D G}\left(\delta^{n+1}, \delta^{n+1}\right) \\
& \geq \frac{1}{2}\left\|\frac{\Delta^{n+1}+\Delta^{n}}{2}\right\|_{a}^{2}-\frac{\kappa_{1} \tau^{2}}{4 \beta H^{2}}\left\|\pi\left(\delta^{n+1}\right)\right\|_{L^{2}(\Omega)}^{2} \\
& =\frac{1}{2}\left\|\frac{\Delta^{n+1}+\Delta^{n}}{2}\right\|_{a}^{2}-\rho^{2}\left\|\pi\left(\delta^{n+1}\right)\right\|_{L^{2}(\Omega)}^{2}
\end{aligned}
$$


For the last term on left hand side of (97), we proceed with the standard procedure with CauchySchwarz inequality to see that

$$
\begin{aligned}
\left\|\pi\left(\delta^{1}\right)\right\|_{L^{2}(\Omega)}^{2} & =\left\|\pi\left(\delta^{0}\right)\right\|_{L^{2}(\Omega)}^{2}+b\left(\delta^{1}-\delta^{0}, \delta^{1}\right)+b\left(\delta^{1}-\delta^{0}, \delta^{0}\right) \\
& \leq\left\|\pi\left(\delta^{0}\right)\right\|_{L^{2}(\Omega)}^{2}+\left\|\pi\left(\delta^{1}-\delta^{0}\right)\right\|_{L^{2}(\Omega)}\left(\left\|\pi\left(\delta^{1}\right)\right\|_{L^{2}(\Omega)}+\left\|\pi\left(\delta^{0}\right)\right\|_{L^{2}(\Omega)}\right) \\
& \leq\left\|\delta^{0}\right\|_{L^{2}(\Omega)}^{2}+2\left\|\delta^{1}-\delta^{0}\right\|_{L^{2}(\Omega)} \max _{0 \leq k \leq N_{T}}\left\|\pi\left(\delta^{k}\right)\right\|_{L^{2}(\Omega)} .
\end{aligned}
$$

Similarly, for the first term on the right hand side of (97), we have

$$
b\left(\delta^{1}-\delta^{0}, \delta^{k+1}+\delta^{k}\right) \leq 2\left\|\delta^{1}-\delta^{0}\right\|_{L^{2}(\Omega)} \max _{0 \leq k \leq N_{T}}\left\|\pi\left(\delta^{k}\right)\right\|_{L^{2}(\Omega)} .
$$

Finally, we estimate the second term on the right hand side of (97) by

$$
\begin{aligned}
& \sum_{k=1}^{n} \sum_{s=1}^{k}\left(r^{s}, \delta^{k+1}+\delta^{k}\right)_{L^{2}(\Omega)} \\
& \quad \leq \sum_{k=1}^{n} R_{1}^{k}\left\|\pi\left(\delta^{k+1}+\delta^{k}\right)\right\|_{L^{2}(\Omega)}+\sum_{k=1}^{n} R_{2}^{k}\left\|(I-\pi)\left(\delta^{k+1}+\delta^{k}\right)\right\|_{L^{2}(\Omega)} \\
& \quad \leq 2\left(\sum_{k=1}^{n} R_{1}^{k}\right) \max _{0 \leq k \leq N_{T}}\left\|\pi\left(\delta^{k}\right)\right\|_{L^{2}(\Omega)}+2\left(\sum_{k=1}^{n} R_{2}^{k}\right) \max _{0 \leq k \leq N_{T}-1}\left\|(I-\pi)\left(\frac{\delta^{k+1}+\delta^{k}}{2}\right)\right\|_{L^{2}(\Omega)} .
\end{aligned}
$$

Combining these estimates, we infer from (97) that

$$
\begin{gathered}
\left(1-\rho^{2}\right)\left\|\pi\left(\delta^{n+1}\right)\right\|_{L^{2}(\Omega)}^{2}+\frac{1}{2}\left\|\frac{\Delta^{n+1}+\Delta^{n}}{2}\right\|_{a}^{2} \\
\leq\left\|\delta^{0}\right\|_{L^{2}(\Omega)}^{2}+2\left((n+1)\left\|\delta^{1}-\delta^{0}\right\|_{L^{2}(\Omega)}+\tau^{2} \sum_{k=1}^{n} R_{1}^{k}\right) \max _{0 \leq k \leq N_{T}}\left\|\pi\left(\delta^{k}\right)\right\|_{L^{2}(\Omega)} \\
+2\left(\tau^{2} \sum_{k=1}^{n} R_{2}^{k}\right) \max _{0 \leq k \leq N_{T}-1}\left\|(I-\pi)\left(\frac{\delta^{k+1}+\delta^{k}}{2}\right)\right\|_{L^{2}(\Omega)} .
\end{gathered}
$$

Using Young's inequality, we have

$$
\begin{aligned}
\max _{0 \leq k \leq N_{T}}\left\|\pi\left(\delta^{k}\right)\right\|_{L^{2}(\Omega)}^{2} & \\
\leq & \frac{2}{1-\rho^{2}}\left\|\delta^{0}\right\|_{L^{2}(\Omega)}^{2}+\frac{8}{\left(1-\rho^{2}\right)^{2}}\left(N_{T}\left\|\delta^{1}-\delta^{0}\right\|_{L^{2}(\Omega)}+\tau^{2} \sum_{k=1}^{N_{T}-1} R_{1}^{k}\right)^{2} \\
& \quad \frac{4}{\left(1-\rho^{2}\right)^{2}}\left(\tau^{2} \sum_{k=1}^{N_{T}-1} R_{2}^{k}\right)^{2}+\max _{0 \leq k \leq N_{T}-1}\left\|(I-\pi)\left(\frac{\delta^{k+1}+\delta^{k}}{2}\right)\right\|_{L^{2}(\Omega)}^{2}
\end{aligned}
$$

which further implies

$$
\begin{aligned}
& \max _{0 \leq k \leq N_{T}-1}\left\|\frac{\delta^{k+1}+\delta^{k}}{2}\right\|_{L^{2}(\Omega)}^{2} \\
& \leq \frac{2}{1-\rho^{2}}\left\|\delta^{0}\right\|_{L^{2}(\Omega)}^{2}+\frac{8}{\left(1-\rho^{2}\right)^{2}}\left(N_{T}\left\|\delta^{1}-\delta^{0}\right\|_{L^{2}(\Omega)}+\tau^{2} \sum_{k=1}^{N_{T}-1} R_{1}^{k}\right)^{2} \\
& \quad+\frac{4}{\left(1-\rho^{2}\right)^{2}}\left(\tau^{2} \sum_{k=1}^{N_{T}-1} R_{2}^{k}\right)^{2}+2 \max _{0 \leq k \leq N_{T}-1}\left\|(I-\pi)\left(\frac{\delta^{k+1}+\delta^{k}}{2}\right)\right\|_{L^{2}(\Omega)}^{2}
\end{aligned}
$$


From the third inequality of Lemma 9, we have

$$
N_{T}\left\|\delta^{1}-\delta^{0}\right\|_{L^{2}(\Omega)} \leq C\left(\Lambda^{-1} H^{2}+\tau^{2}\right) .
$$

Applying the estimates on $R_{1}^{k}$ and $R_{2}^{k}$ in Theorem 10 we have

$$
\tau^{2} \sum_{k=1}^{N_{T}-1}\left(R_{1}^{k}+R_{2}^{k}\right) \leq C\left(\Lambda^{-1} H^{2}+\tau^{2}\right) .
$$

Using (39) and the result of Theorem [10, we have

$$
\max _{0 \leq k \leq N_{T}-1}\left\|(I-\pi)\left(\frac{\delta^{k+1}+\delta^{k}}{2}\right)\right\|_{L^{2}(\Omega)} \leq C\left(\Lambda^{-1} H^{2}+\tau^{2}\right) .
$$

Combining these estimates, we have

$$
\max _{0 \leq k \leq N_{T}-1}\left\|\frac{\delta^{k+1}+\delta^{k}}{2}\right\|_{L^{2}(\Omega)} \leq C\left(\left\|\delta^{0}\right\|_{L^{2}(\Omega)}+\Lambda^{-1} H^{2}+\tau^{2}\right) .
$$

Using a triangle inequality with $\delta^{0}=\theta^{0}-\varepsilon^{0}$, we have

$$
\max _{0 \leq k \leq N_{T}-1}\left\|\frac{\delta^{k+1}+\delta^{k}}{2}\right\|_{L^{2}(\Omega)} \leq C\left(\left\|\varepsilon^{0}\right\|_{L^{2}(\Omega)}+\max _{0 \leq k \leq N_{T}}\left\|\theta^{k}\right\|_{L^{2}(\Omega)}+\Lambda^{-1} H^{2}+\tau^{2}\right) .
$$

Using another triangle inequality with $\varepsilon^{k}=\theta^{k}-\delta^{k}$, we have

$$
\begin{aligned}
\max _{0 \leq k \leq N_{T}-1}\left\|\frac{\varepsilon^{k+1}+\varepsilon^{k}}{2}\right\|_{L^{2}(\Omega)} & \leq \max _{0 \leq k \leq N_{T}-1}\left\|\frac{\delta^{k+1}+\delta^{k}}{2}\right\|_{L^{2}(\Omega)}+\max _{0 \leq k \leq N_{T}}\left\|\theta^{k}\right\|_{L^{2}(\Omega)} \\
& \leq C\left(\left\|\varepsilon^{0}\right\|_{L^{2}(\Omega)}+\max _{0 \leq k \leq N_{T}}\left\|\theta^{k}\right\|_{L^{2}(\Omega)}+\Lambda^{-1} H^{2}+\tau^{2}\right) .
\end{aligned}
$$

Since $u_{H}^{0}$ is the $L^{2}$ projection of $u_{h}^{0}$ onto $V_{H}^{(m)}$, we have

$$
\left\|\varepsilon^{0}\right\|_{L^{2}(\Omega)} \leq\left\|\theta^{0}\right\|_{L^{2}(\Omega)} \leq C \Lambda^{-1} H^{2},
$$

thanks to Lemma 8 The proof is completed by applying the first inequality of Lemma 9

\section{$5 \quad$ Numerical results}

In this section, we will present numerical examples on the scalar wave equation to demonstrate the convergence of our proposed method with respect to the coarse mesh size $H$ and the number of oversampling layers $m$. We take the bulk modulus on the spatial domain $\Omega=[0,1]^{2}$ as part of the Marmousi model as shown in Figure 3 In all the experiments, the IPDG penalty parameter in (3) is set to be $\gamma=4$, which is experimentally sufficient for ensuring the coercivity of the bilinear form $a_{D G}$. The source function $f$ is taken as the Ricker wavelet

$f(t, x, y)=\frac{t-2 / f_{0}}{4 h^{2}} \exp \left(-\pi^{2} f_{0}^{2}\left(t-2 / f_{0}\right)^{2}\right) \exp \left(\frac{(x-0.5)^{2}+(y-0.5)^{2}}{4 h^{2}}\right)$ for all $(t, x, y) \in \Omega_{T}$,

where the fine grid parameter and the central frequency are chosen as $h=1 / 256$ and $f_{0}=20$. Using the fully discrete scheme, we solve for the numerical solution at the final time $T=0.2$ with time step size $\Delta t=10^{-4}$. We compare the coarse-scale approximation with the fine-grid solution. 
The coarse mesh size varies from $H=1 / 64$ to $H=1 / 8$, and the number of oversampling layers varies according with $m \approx 4 \log (1 / H) / \log (1 / 8)$. In all these combinations, we use 4 test basis functions per coarse block to construct the corresponding localized multiscale basis functions.

Table 1 records the error of the final solution It can been observed that the method results in good accuracy and desired convergence in error. Figure 4 depicts the numerical solutions by the fine-scale formulation and the coarse-scale formulation at the final time $T=0.2$. The comparison suggests that our new method provides very good accuracy at a reduced computational expense.

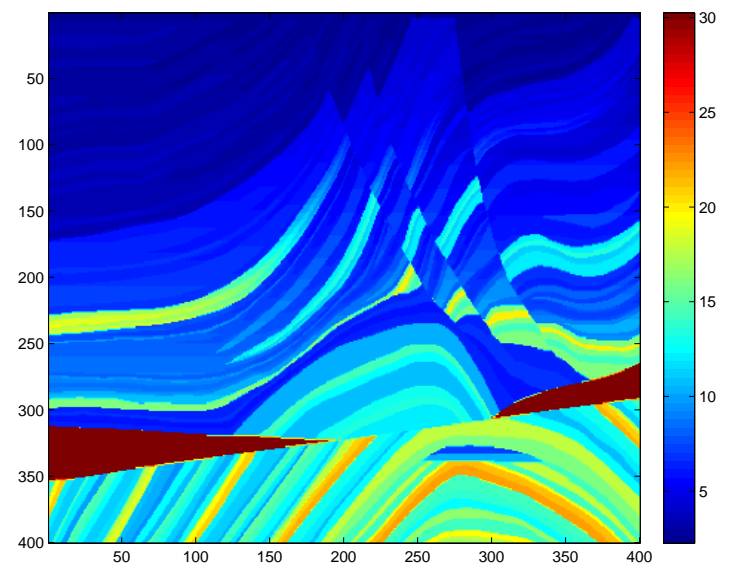

Figure 3: Marmousi model for Experiment 2.

\begin{tabular}{cc|cc}
$m$ & $H$ & Energy error & $L^{2}$ error \\
\hline 4 & $1 / 8$ & $90.0914 \%$ & $64.3121 \%$ \\
6 & $1 / 16$ & $49.1932 \%$ & $26.4195 \%$ \\
7 & $1 / 32$ & $9.9617 \%$ & $4.4368 \%$ \\
8 & $1 / 64$ & $1.1806 \%$ & $0.5049 \%$
\end{tabular}

Table 1: History of convergence for wave propagation in Marmousi model.
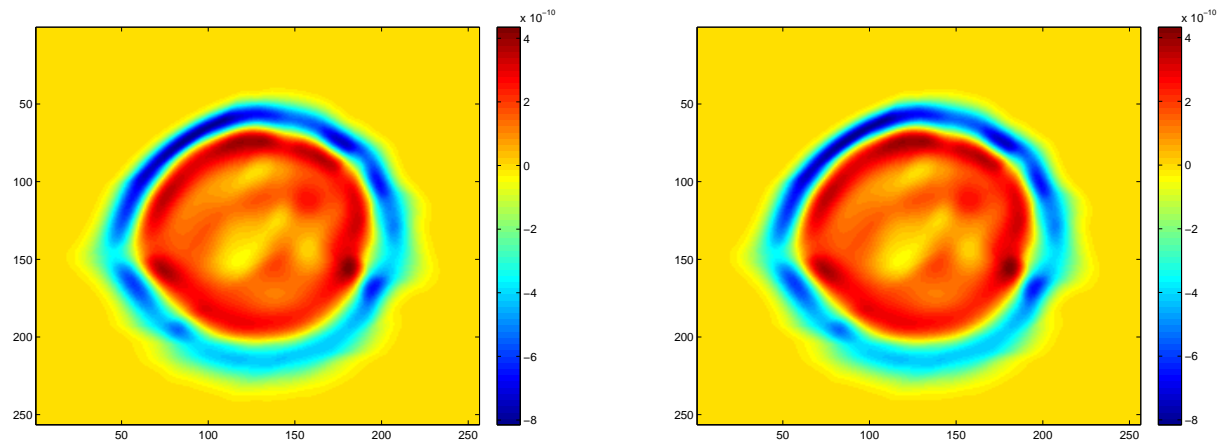

Figure 4: Plots of numerical solution for Experiment 2. Fine solution (left) and multiscale solution (right). 


\section{Conclusion}

In this paper, we present CEM-GMsDGM, a local multiscale model reduction approach in the discontinuous Galerkin framework for the scalar wave equation. The multiscale trial basis functions are defined in coarse oversampled regions by a constraint energy minimization problem, which are in general discontinuous on the coarse grid, and coupled by the IPDG formulation for solving for a coarse-scale approximation. The method is expicit and energy conserving, and exhibits both coarse-mesh convergence and spectral convergence, provided that the oversampling size is appropriately chosen. The stability and the convergence of the method is theoretically analyzed and numerically verified. Numerical results for applying the method on a scalar wave equation are also presented.

\section{Acknowledgements}

The research of Eric Chung is partially supported by the Hong Kong RGC General Research Fund (Project numbers 14304719 and 14302018) and CUHK Faculty of Science Direct Grant 2019-20.

\section{References}

[1] A. Abdulle. On a priori error analysis of fully discrete heterogeneous multiscale fem. SIAM J. Multiscale Modeling and Simulation, 4(2):447-459, 2005.

[2] Assyr Abdulle and Patrick Henning. Multiscale methods for wave problems in heterogeneous media. In Handbook of Numerical Analysis, volume 18, pages 545-576. Elsevier, 2017.

[3] Doghonay Arjmand and Olof Runborg. Analysis of heterogeneous multiscale methods for long time wave propagation problems. Multiscale Modeling $\&$ Simulation, 12(3):1135-1166, 2014 .

[4] A Buffa, TJR Hughes, and G Sangalli. Analysis of a multiscale discontinuous Galerkin method for convection-diffusion problems. SIAM Journal on Numerical Analysis, 44(4):1420-1440, 2006.

[5] Victor Calo, Yalchin Efendiev, and Juan Galvis. A note on variational multiscale methods for high-contrast heterogeneous porous media flows with rough source terms. Advances in water resources, 34(9):1177-1185, 2011.

[6] Z. Chen and T. Y. Hou. A mixed multiscale finite element method for elliptic problems with oscillating coefficients. Mathematics of Computation, 72(242):541-576, 2003.

[7] Siu Wun Cheung, Eric T Chung, Yalchin Efendiev, Wing Tat Leung, and Maria Vasilyeva. Constraint energy minimizing generalized multiscale finite element method for dual continuum model. arXiv preprint arXiv:180\%.10955, 2018.

[8] Siu Wun Cheung, Eric T Chung, and Wing Tat Leung. Constraint energy minimizing generalized multiscale discontinuous galerkin method. arXiv preprint arXiv:1909.12461, 2019.

[9] Siu Wun Cheung and Nilabja Guha. Dynamic data-driven bayesian gmsfem. Journal of Computational and Applied Mathematics, 353:72 - 85, 2019.

[10] C.C. Chu, I.G. Graham, and T. Hou. A new multiscale finite element methods for highcontrast elliptic interface problem. Mathematics of Computation, 79:1915-1955, 2010. 
[11] Eric Chung, Yalchin Efendiev, and Thomas Y Hou. Adaptive multiscale model reduction with generalized multiscale finite element methods. Journal of Computational Physics, 320:69-95, 2016.

[12] Eric Chung, Yalchin Efendiev, and Wing Tat Leung. Constraint energy minimizing generalized multiscale finite element method in the mixed formulation. Computational Geosciences, $22(3): 677-693,2018$.

[13] Eric T Chung, Yalchin Efendiev, Richard L Gibson, and Wing Tat Leung. Residual-driven online multiscale methods for acoustic-wave propagation in 2d heterogeneous media. Geophysics, 82(2):T69-T77, 2017.

[14] Eric T Chung, Yalchin Efendiev, Richard L Gibson Jr, and Maria Vasilyeva. A generalized multiscale finite element method for elastic wave propagation in fractured media. GEMInternational Journal on Geomathematics, pages 1-20, 2015.

[15] Eric T Chung, Yalchin Efendiev, and Wing Tat Leung. Generalized multiscale finite element methods for wave propagation in heterogeneous media. Multiscale Modeling 85 Simulation, 12(4):1691-1721, 2014.

[16] Eric T Chung, Yalchin Efendiev, and Wing Tat Leung. Residual-driven online generalized multiscale finite element methods. Journal of Computational Physics, 302:176-190, 2015.

[17] Eric T. Chung, Yalchin Efendiev, and Wing Tat Leung. An online generalized multiscale discontinuous galerkin method (gmsdgm) for flows in heterogeneous media. Communications in Computational Physics, 21(2):401422, 2017.

[18] Eric T Chung, Yalchin Efendiev, and Wing Tat Leung. Constraint energy minimizing generalized multiscale finite element method. Computer Methods in Applied Mechanics and Engineering, 339:298-319, 2018.

[19] Eric T Chung and Wing Tat Leung. Mixed gmsfem for the simulation of waves in highly heterogeneous media. Journal of Computational and Applied Mathematics, 306:69-86, 2016.

[20] E.T. Chung, Y. Efendiev, and W.T. Leung. An adaptive generalized multiscale discontinuous galerkin method for high-contrast flow problems. SIAM Multiscale Modeling and Simulation, 16(3):1227-1257, 2018.

[21] ET Chung, Y Efendiev, and G Li. An adaptive GMsFEM for high-contrast flow problems. Journal of Computational Physics, 273:54-76, 2014.

[22] W. E and B. Engquist. Heterogeneous multiscale methods. Comm. Math. Sci., 1(1):87-132, 2003.

[23] W. E, P. Ming, and P. Zhang. Analysis of the heterogeneous multiscale method for elliptic homogenization problems. J. Amer. Math. Soc., 18(1):121-156, 2005.

[24] Y. Efendiev, J. Galvis, and T. Hou. Generalized multiscale finite element methods. Journal of Computational Physics, 251:116-135, 2013.

[25] Y Efendiev, J Galvis, R Lazarov, M Moon, and M Sarkis. Generalized multiscale finite element method. Symmetric interior penalty coupling. Journal of Computational Physics, $255: 1-15,2013$.

[26] Y Efendiev, J Galvis, G Li, and M Presho. Generalized multiscale finite element methods. Oversampling strategies. International Journal for Multiscale Computational Engineering, accepted, 2013. 
[27] Y. Efendiev, J. Galvis, and X.H. Wu. Multiscale finite element methods for high-contrast problems using local spectral basis functions. Journal of Computational Physics, 230:937$955,2011$.

[28] Y. Efendiev and T. Hou. Multiscale Finite Element Methods: Theory and Applications. Springer, 2009.

[29] Y. Efendiev, T. Hou, and X.H. Wu. Convergence of a nonconforming multiscale finite element method. SIAM J. Numer. Anal., 37:888-910, 2000.

[30] Yalchin Efendiev, Raytcho Lazarov, Minam Moon, and Ke Shi. A spectral multiscale hybridizable discontinuous Galerkin method for second order elliptic problems. Computer Methods in Applied Mechanics and Engineering, 292:243-256, 2015.

[31] Yalchin Efendiev, Wing Tat Leung, S. W. Cheung, N. Guha, V. H. Hoang, and B. Mallick. Bayesian multiscale finite element methods. modeling missing subgrid information probabilistically. International Journal for Multiscale Computational Engineering, 15(2):175-197, 2017.

[32] D. Elfverson, E. Georgoulis, A. Mlqvist, and D. Peterseim. Convergence of a discontinuous galerkin multiscale method. SIAM Journal on Numerical Analysis, 51(6):3351-3372, 2013.

[33] Björn Engquist, Henrik Holst, and Olof Runborg. Multiscale methods for wave propagation in heterogeneous media over long time. In Numerical analysis of multiscale computations, pages 167-186. Springer, 2012.

[34] T. Hou and X.H. Wu. A multiscale finite element method for elliptic problems in composite materials and porous media. J. Comput. Phys., 134:169-189, 1997.

[35] Thomas Y Hou and Pengchuan Zhang. Sparse operator compression of higher-order elliptic operators with rough coefficients. Research in the Mathematical Sciences, 4(1):24, 2017.

[36] T.J.R. Hughes, G.R. Feijóo, L. Mazzei, and J.-B. Quincy. The variational multiscale method - a paradigm for computational mechanics. Comput. Methods Appl. Mech Engrg., 127:3-24, 1998.

[37] TJR Hughes and G Sangalli. Variational multiscale analysis: the fine-scale Green's function, projection, optimization, localization, and stabilized methods. SIAM Journal on Numerical Analysis, 45(2):539-557, 2007.

[38] O. Iliev, R. Lazarov, and J. Willems. Variational multiscale finite element method for flows in highly porous media. Multiscale Model. Simul., 9(4):1350-1372, 2011.

[39] Lijian Jiang and Yalchin Efendiev. A priori estimates for two multiscale finite element methods using multiple global fields to wave equations. Numerical Methods for Partial Differential Equations, 28(6):1869-1892, 2012.

[40] Roland Maier and Daniel Peterseim. Explicit computational wave propagation in microheterogeneous media. BIT Numerical Mathematics, 59(2):443-462, 2019.

[41] Axel Målqvist and Daniel Peterseim. Localization of elliptic multiscale problems. Mathematics of Computation, 83(290):2583-2603, 2014.

[42] Houman Owhadi. Multigrid with rough coefficients and multiresolution operator decomposition from hierarchical information games. SIAM Review, 59(1):99-149, 2017. 
[43] Houman Owhadi and Lei Zhang. Numerical homogenization of the acoustic wave equations with a continuum of scales. Computer Methods in Applied Mechanics and Engineering, 198(3-4):397-406, 2008.

[44] Houman Owhadi, Lei Zhang, and Leonid Berlyand. Polyharmonic homogenization, rough polyharmonic splines and sparse super-localization. ESAIM: Mathematical Modelling and Numerical Analysis, 48(2):517-552, 2014.

[45] G Papanicolau, A Bensoussan, and J-L Lions. Asymptotic analysis for periodic structures. Elsevier, 1978.

[46] Jun Sur Richard Park, Siu Wun Cheung, Tina Mai, and Viet Ha Hoang. Multiscale simulations for upscaled multi-continuum flows. arXiv preprint arXiv:1909.04722, 2019.

[47] Béatrice Rivière. Discontinuous Galerkin methods for solving elliptic and parabolic equations: theory and implementation. Society for Industrial and Applied Mathematics, 2008.

[48] Maria Vasilyeva, Eric T Chung, Siu Wun Cheung, Yating Wang, and Georgy Prokopev. Nonlocal multicontinua upscaling for multicontinua flow problems in fractured porous media. Journal of Computational \&3 Applied Mathematics, 355:258-267, 2019.

[49] Tetyana Vdovina, Susan E Minkoff, and Oksana Korostyshevskaya. Operator upscaling for the acoustic wave equation. Multiscale Modeling ES Simulation, 4(4):1305-1338, 2005.

[50] Min Wang, Siu Wun Cheung, Eric T. Chung, Maria Vasilyeva, and Yuhe Wang. Generalized multiscale multicontinuum model for fractured vuggy carbonate reservoirs. Journal of Computational and Applied Mathematics, 366:112370, 2020.

[51] X.H. Wu, Y. Efendiev, and T.Y. Hou. Analysis of upscaling absolute permeability. Discrete and Continuous Dynamical Systems, Series B., 2:158-204, 2002. 